\section{Cureus}

Received 05/12/2019

Review began 05/15/2019

Review ended 05/18/2019

Published 05/23/2019

\section{(c) Copyright 2019}

Mesko et al. This is an open access article distributed under the terms of the Creative Commons Attribution License CC-BY 3.0., which permits unrestricted use, distribution, and reproduction in any medium, provided the original author and source are credited.

\title{
Spine Stereotactic Radiosurgery for Metastatic Pheochromocytoma
}

Shane Mesko ${ }^{1}$, Brian J. Deegan ${ }^{1}$, Neil M. D'Souza ${ }^{2}$, Amol J. Ghia ${ }^{1}$, Bhavana V. Chapman ${ }^{1}$, Behrang Amini ${ }^{3}$, Mary Frances McAleer ${ }^{1}$, Xin A. Wang ${ }^{4}$, Paul D. Brown ${ }^{5}$, Claudio E. Tatsui ${ }^{6}$ , Laurence Rhines ${ }^{6}$, Jing $\mathrm{Li}^{1}$

1. Radiation Oncology, The University of Texas MD Anderson Cancer Center, Houston, USA 2. Radiation Oncology, Mays Cancer Center, University of Texas, San Antonio, USA 3. Radiology, The University of Texas MD Anderson Cancer Center, Houston, USA 4. Radiation Physics, The University of Texas MD Anderson Cancer Center, Houston, USA 5. Radiation Oncology, Mayo Clinic, Rochester, USA 6. Neurosurgery, The University of Texas MD Anderson Cancer Center, Houston, USA

$\square$ Corresponding author: Shane Mesko, smesko@mdanderson.org Disclosures can be found in Additional Information at the end of the article

\section{Abstract}

Purpose: Despite aggressive primary treatment, up to $13.5 \%$ of patients diagnosed with pheochromocytoma may develop metastases, most often affecting the axial skeleton. Given that systemic therapy options are often inadequate, local therapy remains the cornerstone of palliation for these patients. Historically poor responses to standard fractionated radiotherapy have led to the consideration of stereotactic radiosurgery as an option to overcome potential radioresistance and provide durable local control of these tumors. Here we report our institutional experience in treating spine metastases from pheochromocytoma with spine stereotactic radiosurgery (SSRS).

Methods and materials: Our clinical databases were retrospectively reviewed for patients with metastatic pheochromocytoma treated with SSRS from 2000-2017. Seven patients with 16 treated metastatic spinal lesions were identified. Local control was evaluated using magnetic resonance imaging (MRI). Pain and symptom data were assessed to evaluate toxicity using Common Terminology Criteria for Adverse Events (CTCAE) v4.03. The Kaplan-Meier method was used to assess local control and overall survival (OS).

Results: Median follow-up for treated lesions was 11 months (range 2.2 - 70.8). Most lesions were treated to a dose of $27 \mathrm{~Gy}$ in three fractions (62.5\%). Other fractionation schemes included $24 \mathrm{~Gy}$ in one fraction (25\%), $16 \mathrm{~Gy}$ in one fraction (6.3\%), and $18 \mathrm{~Gy}$ in three fractions (6.3\%). Treatment sites included the cervical spine (18.8\%), thoracic spine (37.5\%), lumbar spine (31.3\%), and sacrum (12.5\%). The crude local control rate was $93.7 \%$, with one thoracic spine lesion progressing 20.7 months after treatment with $24 \mathrm{~Gy}$ in one fraction. Kaplan-Meier OS rates at 1 and 2 years after SSRS were $71.4 \%$ and $42.9 \%$, respectively. Most common toxicities included acute grade 1-2 pain and fatigue. There was one case of vertebral fracture in a cervical spine lesion treated to $27 \mathrm{~Gy}$ in three fractions, which was managed non-surgically.

Conclusion: Very few studies have explored the use of SSRS in metastatic pheochromocytoma. Our data suggest this modern radiation modality is effective, safe, and provides durable local control to palliate symptoms and potentially limit further metastatic seeding. Larger patient numbers and longer follow-up will further define the role of SSRS as a treatment option in these patients. 
Categories: Radiation Oncology, Oncology

Keywords: ssrs, stereotactic radiation, pheochromocytoma, spine stereotactic radiosurgery, metastatic pheochromocytoma

\section{Introduction}

Pheochromocytoma is a catecholamine-secreting tumor originating from chromaffin cells in the adrenal medulla and belongs to the intra-adrenal subcategory of paragangliomas (PGLs) [12]. The classic presenting triad is episodic headache, sweating, and palpitations in the context of persistent hypertension [3]. Pheochromocytomas are rare with only 1-6 cases per million people per year, and approximately $13.5 \%$ of these are found to be metastatic [3-5].

Five-year survival for metastatic disease ranges from 34\% to 60\%, depending largely on organ involvement, compared to $90 \%$ to $95 \%$ survival rates for benign disease [6]. Bone metastases carry the most favorable prognosis while visceral metastases portend comparatively poor survival [7-9]. Surgery remains the mainstay of therapy for both benign and malignant disease. However, recurrence rates for metastatic disease are high even after resection, and the goals of therapy are often geared toward palliation of symptoms [10]. Iodine ${ }^{131}$-labeled metaiodobenzylguanidine ( $\left.{ }^{131}-\mathrm{MIBG}\right)$ has also shown utility as an adjunct to surgery $[9,11]$. Systemic therapy, including chemotherapy with cyclophosphamide, vincristine, and dacarbazine (CVD), has shown short-lived benefit, while targeted therapies directed against vascular endothelial growth factor (VEGF) and platelet-derived growth factor receptors (PDGF$\mathrm{R})$ are under investigation $[2,12]$.

The role of radiotherapy in pheochromocytoma is poorly defined. Although external beam radiation is commonly utilized in head and neck parasympathetic PGLs, evidence for benefit in pheochromocytoma is poor. A 1978 review on early reports of external radiotherapy in metastatic pheochromocytoma concluded that pheochromocytoma is most likely a radiationresistant tumor, but that radiotherapy may be beneficial for symptom control in bone and lymph node metastasis when delivered at high doses [13]. Due to the rarity of malignant pheochromocytoma, studies with sizable cohorts are difficult to assemble and are consequently scarce, particularly in the radiation literature. Radiotherapy advances, especially with respect to image guidance, intensity modulated radiation therapy (IMRT), and spine stereotactic radiosurgery (SSRS), could prove valuable in the treatment of malignant pheochromocytoma [14-15]. SSRS, in particular, is an attractive option to overcome traditional radioresistance and provide more durable local control of metastatic pheochromocytoma. The purpose of this series is to report our institutional experience using SSRS to treat spine metastases from pheochromocytoma in an effort to provide evidence of the viability and benefit of this option in these rare cases.

\section{Materials And Methods}

\section{Study design and patients}

The available records of patients with histologically confirmed metastatic pheochromocytoma treated with SSRS between 2000 and 2017 were retrospectively reviewed in an institutional review board (IRB) approved analysis.

\section{Data sources}

Patient demographics, treatment date(s), location, and dose prescription information were sourced from institutional databases. Histological confirmation, oncologic history, clinical surveillance, toxicity outcomes, and survival data were obtained via review of the medical records. 


\section{Treatment}

Patients were treated using IMRT with multi-modality image guidance. The details of patient setup, treatment planning, and delivery have been previously described [15-16]. In short, patients were immobilized using a full body vacuum cradle (BlueBAG, Elekta, Stockholm, Sweden). In addition, a thermoplastic mask (High Precision System for Head, Neck and Shoulders, Orfit Industries America, Wijnegem, Belgium) or plastic body cover sheet (BodyFIX, Elekta, Stockholm, Sweden) was added for precise patient positioning and immobilization. Spine magnetic resonance imaging (MRI) was used for target and critical structure delineation. Images were fused to simulation computed tomography (CT) scan for dose calculation and IMRT planning. The IMRT plan was optimized in Pinnacle treatment planning software (Version 9.8 or 9.10, Philips Radiation Oncology Systems, Andover, MA) using 7 to 9 posterior beams with step-and-shoot technique. Multi-modality image guidance was achieved with either CTon-rails, Varian on-board imaging system (Varian Medical Systems, Palo Alto, CA), and/or Brainlab ExacTrac targeting system (Brainlab AG, Feldkirchen, Germany). Alignment was assessed by the treating radiation oncologist. Stereoscopic imaging (ExacTrac) was used to monitor and adjust intra-fractional patient motion. A dedicated radiation physicist was also present to oversee the work flow and perform the alignment.

Treatment was prescribed to the gross tumor volume (GTV) which was defined as all radiographically evident tumor including bony, paraspinal soft tissue, and epidural disease as based on image-fusion data sets. The clinical treatment volume (CTV) included the GTV plus an additional expansion to include adjacent contiguous bone marrow at risk per the International Spine Radiosurgery Consortium guidelines [17]. The GTVs were prescribed to receive either 18 Gy or $27 \mathrm{~Gy}$ in three fractions under the multifraction protocol, while the single fraction treatments consisted of either $24 \mathrm{~Gy}$ or $18 \mathrm{~Gy}$. SSRS was not given as a boost after conventional radiation in any case. Spinal cord and cauda equina were contoured as true structures rather than thecal sac and no planning target volume (PTV) was used. Dose constraints for normal structures per our institutional standard depending on fractionation and prior treatment [14]. Multi-fraction treatments were delivered on consecutive or alternating days depending on machine availability and patient convenience.

\section{Outcomes and assessment}

Follow-up for the cohort consisted of MRI and clinical exam every three months for the first two years, then every 3-6 months thereafter. Patients could have had additional surveillance imaging performed at the discretion of the treating physician. End points included local control, overall survival, and toxicity. Local recurrence was evaluated with spine MRI, interpreted by the reading radiologist, and confirmed by the radiation oncologist in the context of the original SSRS treatment plan. Pain and symptom data were assessed to evaluate toxicity and were scored using the Common Terminology Criteria for Adverse Events (CTCAE) version 4.03. Toxicity was recorded if radiation therapy was a possible cause, but excluded if attributed to disease progression. Local control and overall survival (OS) were analyzed using the KaplanMeier method.

\section{Results}

\section{Patient and treatment characteristics}

Baseline patient characteristics are listed in Table 1. Ultimately, seven patients with a total of 16 treated lesions were deemed suitable for retrospective analysis. The median age at the time of SSRS was 58 years (range: 46 - 68 years). All patients were of good performance status at the time of radiation treatment with a median Karnofsky Performance Status (KPS) of 90. Two patients presented with metastatic disease at the time of initial diagnosis, while the other five had a median time to first metastasis of 62.9 months (range 19.3-178) (Table 2). All patients in 


\section{Cureus}

the series had undergone upfront surgical resection of their primary disease and had received systemic chemotherapy and exhibited systemically progressive disease at the time of SSRS.

\begin{tabular}{|c|c|c|c|c|}
\hline Patient & Age & Sex & KPS & Lesions treated \\
\hline 1 & 63 & Male & 80 & 1 \\
\hline 2 & 46 & Male & 90 & 5 \\
\hline 3 & 60 & Male & 100 & 4 \\
\hline 4 & 50 & Male & 80 & 1 \\
\hline 5 & 56 & Female & 90 & 2 \\
\hline 6 & 68 & Male & 90 & 2 \\
\hline 7 & 58 & Male & 90 & 1 \\
\hline
\end{tabular}

\section{TABLE 1: Patient characteristics}

KPS: Karnofsky Performance Status

\begin{tabular}{|c|c|c|c|c|c|}
\hline Patient & $\begin{array}{l}\text { Time to SSRS from } \\
\text { Metastasis (months) }\end{array}$ & $\begin{array}{l}\text { Vital } \\
\text { Status }\end{array}$ & $\begin{array}{l}\text { Total Survival from } \\
\text { Metastasis (months) }\end{array}$ & $\begin{array}{l}\text { Survival from } \\
\text { SSRS (months) }\end{array}$ & Toxicity (grade) \\
\hline 1 & 25.4 & Deceased & 36.4 & 11.0 & Pain (2), Fatigue (1) \\
\hline 2 & 9.1 & Deceased & 85.8 & 76.7 & None \\
\hline 3 & 72.7 & Deceased & 86.1 & 13.4 & $\begin{array}{l}\text { C5 Fracture (2), Pain } \\
\text { (2), Fatigue (1) }\end{array}$ \\
\hline 4 & 9.8 & Deceased & 39.4 & 29.6 & None \\
\hline 5 & 13.3 & Deceased & 16.5 & 3.2 & None \\
\hline 6 & 19.5 & Deceased & 43.3 & 23.8 & None \\
\hline 7 & 6.8 & Alive & 48.2 & 41.1 & Pain (2) \\
\hline
\end{tabular}

\section{TABLE 2: Patient survival and toxicity}

SSRS: Spine stereotactic radiosurgery

The most common SSRS fractionation scheme was $27 \mathrm{~Gy}$ in three fractions (62.5\%). Other fractionation schemes included $24 \mathrm{~Gy}$ in one fraction (25\%), $16 \mathrm{~Gy}$ in one fraction (6.3\%) and $18 \mathrm{~Gy}$ in three fractions (6.3\%). Treatment sites included the cervical-spine (18.8\%), thoracicspine (37.5\%), lumbar-spine (31.3\%) and sacrum (12.5\%) (Table 3). The median number of 


\section{Cureus}

vertebral levels treated per course was two (range 1-3).

\begin{tabular}{|c|c|c|c|c|}
\hline Patient & Treatment Area & Dose and Fractionation & Local Control & Freedom from Failure (months) \\
\hline 1 & $\mathrm{C} 5-\mathrm{C} 7$ & 27 Gy (9 Gy x 3) & Yes & 6.4 \\
\hline \multirow{5}{*}{2} & L2 & 16 Gy (16 Gy x 1) & Yes & 65.1 \\
\hline & L1, L3 & 27 Gy (9 Gy x 3) & Yes & 25.3 \\
\hline & T8-T10 & 24 Gy (24 Gy x 1) & No & 20.7 \\
\hline & T1 & 24 Gy (24 Gy x 1) & Yes & 3.6 \\
\hline & T11-T12 & 27 Gy (9 Gy x 3) & Yes & 3.6 \\
\hline \multirow{4}{*}{3} & C5 & 27 Gy (9 Gy x 3) & Yes & 11.0 \\
\hline & $\mathrm{T} 7$ & 27 Gy (9 Gy x 3) & Yes & 11.0 \\
\hline & 15 & 21 Gy (9 Gy x 3) & Yes & 10.1 \\
\hline & S1-S2 & 18 Gy (6 Gy x 3) & Yes & 10.7 \\
\hline 4 & L3 & 24 Gy (24 Gy x 1) & Yes & 25.4 \\
\hline \multirow{2}{*}{5} & L4-15 & 27 Gy (9 Gy x 3) & Yes & 2.3 \\
\hline & T3-T5 & 27 Gy (9 Gy x 3) & Yes & 2.2 \\
\hline \multirow{2}{*}{6} & S3-S5 & 27 Gy (9 Gy x 3) & Yes & 20.1 \\
\hline & C1 & 27 Gy (9 Gy x 3) & Yes & 16.8 \\
\hline$r$ & L3-L4 & 24 Gy (24 Gy x 1) & Yes & 37.6 \\
\hline
\end{tabular}

TABLE 3: Spine stereotactic radiosurgery (SSRS) treatment regimens, sites, and local control

\section{Tumor control}

Median follow-up for each treated site was 11 months (range: 2 - 71 months) (Table 3). Crude local control rate was $93.7 \%$ with one treatment failure observed during the follow-up period. This solitary local failure occurred 21 months post-treatment in a patient treated with 24 Gy in one fraction. Six of the seven patients treated died during the course of follow-up. and no deaths were considered treatment related. Kaplan-Meier OS at one and two years after SSRS were $71.4 \%$ and $42.9 \%$, respectively.

\section{Toxicity}

Toxicity in this cohort was minimal and categorically low grade (CTCAE grade 2 or lower; Table 2). There were three cases of grade 2 non-radicular, musculoskeletal-type pain. Two patients experienced grade 1 fatigue after treatment and one patient developed a grade 2 vertebral compression fracture at the level of C5, which was managed non-operatively. Overall, 
treatment was well tolerated with little associated morbidity.

\section{Discussion}

The traditional treatment modalities for metastatic pheochromocytoma consist of surgical resection or debulking in conjunction with $\mathrm{I}^{131}$-MIBG. Combinations of CVD triplet chemotherapy are also added in some cases [18]. The role of external beam radiation therapy (EBRT) has been scarcely examined and remains a subject of debate. PGLs as a whole are typically considered slowly proliferating tumors and as such, the efficacy of radiotherapy, in comparison to surgery, is best assessed by radiographic evolution and symptomatic responses rather than the degree of tumor regression [19]. While recognizing the role of radiotherapy in PGLs of the head and neck is well established - a review of more than 1000 tumors reported near $90 \%$ local control rate at 10-year follow with a total dose of 40 to $45 \mathrm{~Gy}$ - the role of radiation in non-head and neck PGLs is less clear, particularly in the metastatic setting [20]. An influential 1978 review by Drasin examined case reports describing EBRT in metastatic pheochromocytoma, finding the evidence to be lacking in support of EBRT for treatment of the primary tumor, but more favorable for palliative treatment of bony metastases [13]. Although the Drasin review suggested that higher doses may be more efficacious, high-dose EBRT utilizing older technology has been associated with considerable toxicity, especially in the retroperitoneum [21]. Various case reports have since described the effectiveness of modern high-dose radiotherapy in the treatment of metastatic pheochromocytoma to the urinary bladder, liver, lymph nodes, and lumbar spine [22-25]. A recent review of 17 patients with metastases of pheochromocytoma in the thorax, abdomen, or pelvis who were treated with a variety of modern radiotherapy techniques, including IMRT, showed EBRT to be effective for local control and symptomatic relief in $76 \%$ of patients especially in conjunction with $\mathrm{I}^{131-} \mathrm{MIBG}$ in cases of bulky tumors [26].

In this study, we describe the promising role of a modern modality in radiation therapy, stereotactic radiosurgery (SRS), in the management of metastatic pheochromocytoma to the axial skeleton. A local control rate of $93.5 \%$ was achieved for these treated segments using a variety of fractionation schemes. In the recent past, SRS has been successfully applied to the management of jugular PGLs, contributing to disease stabilization and even regression [19,2728]. In contrast, the use of SSRS in the treatment of malignant pheochromocytoma is a relatively unexplored entity. A recent retrospective review by Vogel et al. in 2014 compiled together 24 patients with malignant pheochromocytoma and PGL who were treated with various forms of EBRT [6]. Of these patients, only one was treated with SSRS to bony metastases, specifically to C3 at a dose of $24 \mathrm{~Gy}$, and two others received SRS for metastases to the liver and the organ of Zuckerkandl. As a group, these three patients treated with SRS achieved more durable local control in comparison to patients receiving standard fractionation. Our results corroborate these findings and further point the effectiveness in terms of local control of SRSS in treatment of spine metastases in particular. With respect to the lone treatment failure seen in our sample, this occurred in the context of florid systemic progression 21 months after treatment, and may represent re-seeding rather than SSRS failure.

Toxicities in our study were limited to grade 2 . Three out of six patients experienced some form of treatment-related toxicity, with both pain and fatigue (grade 2 and grade 1, respectively) occurring most frequently. All three patients who received SSRS within 1 year of the development of metastasis survived beyond two years post-treatment. These results suggest there may be a benefit to early therapeutic intervention with SSRS in both local control and overall disease progression.

Limitations of this study include the relatively small number of cases which effectively precludes extensive statistical analysis. These limitations are unsurprising given the rarity of 
this condition and the limited utilization of radiotherapy in the overall management of pheochromocytoma. Future studies may better define the long-term toxicities of treatment, the durability of local control, and optimal timing/context for integration of radiation therapy.

\section{Conclusions}

To our knowledge, this is one of the first studies describing the utility of SSRS in the treatment of metastatic pheochromocytoma. Our data suggest that SSRS is an effective, safe, and durable treatment option. Despite strong local control, pheochromocytoma remains a progressive, metastatic disease requiring improvements in systemic therapy. Given the robust tumor control of SSRS and the possibility for metastatic foci to serve as sources for further systemic spread, proactive treatment of spinal metastasis earlier in the disease course may offer therapeutic benefit to these patients. Larger patient numbers and longer follow-up is required to address this issue in the future.

\section{Additional Information}

\section{Disclosures}

Human subjects: Consent was obtained by all participants in this study. MD Anderson Institutional Review Board issued approval PA13-0218. This was an IRB-approved retrospective study. Animal subjects: All authors have confirmed that this study did not involve animal subjects or tissue. Conflicts of interest: In compliance with the ICMJE uniform disclosure form, all authors declare the following: Payment/services info: All authors have declared that no financial support was received from any organization for the submitted work. Financial relationships: All authors have declared that they have no financial relationships at present or within the previous three years with any organizations that might have an interest in the submitted work. Other relationships: Dr. Shane Mesko has a consulting agreement with Oscar Healthcare, outside the submitted work. Dr. Jing Li has received research funding from Medtronic to support a different clinical trial on SSRS, outside the submitted work Dr. Paul Brown has received personal fees from UpToDate (contributor) and Novella Clinical (DMSB Member), outside the submitted work. .

\section{References}

1. Adler JT, Meyer-Rochow GY, Chen H, Benn DE, Robinson BG, Sippel RS, Sidhu SB: Pheochromocytoma: current approaches and future directions . Oncologist. 2008, 13:779-793. 10.1634/theoncologist.2008-0043

2. Grogan RH, Mitmaker EJ, Duh QY: Changing paradigms in the treatment of malignant pheochromocytoma. Cancer Control. 2011, 18:104-112. 10.1177/107327481101800205

3. Manger WM: The vagaries of pheochromocytomas. Am J Hypertens. 2005, 18:1266 -1270. 10.1016/j.amjhyper.2005.06.026

4. Goldstein RE, O'neill JA, Holcomb GW, et al.: Clinical experience over 48 years with pheochromocytoma. Ann Surg. 1999, 229:755-764. 10.1097/00000658-199906000-00001

5. Scholz T, Eisenhofer G, Pacak K, Dralle H, Lehnert H: Clinical review: current treatment of malignant pheochromocytoma. J Clin Endocrinol Metab. 2007, 92:1217-1225. 10.1210/jc.20061544

6. Vogel J, Atanacio AS, Prodanov T, et al.: External beam radiation therapy in treatment of malignant pheochromocytoma and paraganglioma. Front Oncol. 2014, 4:166. 10.3389/fonc. 2014.00166

7. John H, Ziegler WH, Hauri D, Jaeger P: Pheochromocytomas: can malignant potential be predicted?. Urology. 1999, 53:679-683. 10.1016/S0090-4295(98)00612-8

8. Mundschenk J, Lehnert H: Malignant pheochromocytoma. Exp Clin Endocrinol Diabetes. 1998, 106:373-376. 10.1055/s-0029-1212001

9. Pacak K, Eisenhofer G, Ahlman H, et al.: Pheochromocytoma: recommendations for clinical practice from the First International Symposium October 2005. Nat Clin Pract Endocrinol 
Metab. 2007, 3:92-102. 10.1038/ncpendmet0396

10. Sisson JC, Shulkin BL, Esfandiari NH: Courses of malignant pheochromocytoma: implications for therapy. Ann N Y Acad Sci. 2006, 1073:505-511. 10.1196/annals.1353.053

11. Sisson JC, Shapiro B, Shulkin BL, Urba S, Zempel S, Spaulding S.: Treatment of malignant pheochromocytomas with 131-I metaiodobenzylguanidine and chemotherapy. Am J Clin Oncol. 1999, 22:364-370.

12. Averbuch SD, Steakley CS, Young RC, Gelmann EP, Goldstein DS, Stull R, Keiser HR: Malignant pheochromocytoma: effective treatment with a combination of cyclophosphamide, vincristine, and dacarbazine. Ann Intern Med. 1988, 109:267-273. 10.7326/0003-4819-109-4267

13. Drasin H: Treatment of malignant pheochromocytoma. West J Med. 1978, 128:106-111.

14. Ghia AJ, Guha-thakurta N, Hess K, et al.: Phase 1 study of spinal cord constraint relaxation with single session spine stereotactic radiosurgery in the primary management of patients with inoperable, previously unirradiated metastatic epidural spinal cord compression. Int J Radiat Oncol Biol Phys. 2018, 102:1481-1488. 10.1016/j.ijrobp.2018.07.2023

15. Chang EL, Shiu AS, Lii MF, et al.: Phase I clinical evaluation of near-simultaneous computed tomographic image-guided stereotactic body radiotherapy for spinal metastases. Int J Radiat Oncol Biol Phys. 2004, 59:1288-1294. 10.1016/j.ijrobp.2004.04.025

16. Wang X, Zhao Z, Luo D, et al.: Submillimeter alignment of more than three contiguous vertebrae in spinal SRS/SBRT with 6-degree couch. J Appl Clin Med Phys. 2017, 18:225-236. 10.1002/acm2.12153

17. Cox BW, Spratt DE, Lovelock M, et al.: International Spine Radiosurgery Consortium consensus guidelines for target volume definition in spinal stereotactic radiosurgery. Int J Radiat Oncol Biol Phys. 2012, 83:597-605. 10.1016/j.ijrobp.2012.03.009

18. Andersen KF, Altaf R, Krarup-Hansen A, Kromann-Andersen B, Horn T, Christensen NJ, Hendel HW: Malignant pheochromocytomas and paragangliomas - the importance of a multidisciplinary approach. Cancer Treat Rev. 2011, 37:111-9. 10.1016/j.ctrv.2010.07.002

19. Kupferman ME, Hanna EY: Paragangliomas of the head and neck . Curr Oncol Rep. 2008, 10:156-161. 10.1007/s11912-008-0024-9

20. Hu K, Persky MS: The multidisciplinary management of paragangliomas of the head and neck, part 2. Oncology. 2003, 17:1143-1161.

21. Krych AJ, Foote RL, Brown PD, Garces YI, Link MJ: Long-term results of irradiation for paraganglioma. Int J Radiat Oncol Biol Phys. 2006, 65:1063-1066. 10.1016/j.ijrobp.2006.02.020

22. Yoshida S, Nakagomi K, Goto S, Kobayashi S: Malignant pheochromocytoma of the urinary bladder effectiveness of radiotherapy in conjunction with chemotherapy. Int J Urol. 2004, 11:175-7. 10.1111/j.1442-2042.2003.00758.x

23. Yu L, Fleckman AM, Chadha M, Sacks E, Levetan C, Vikram B: Radiation therapy of metastatic pheochromocytoma: case report and review of the literature. Am J Clin Oncol. 1996, 19:38993.

24. Siddiqui MZ, Von Eyben FE, Spanos G: High-voltage irradiation and combination chemotherapy for malignant pheochromocytoma. Cancer. 1988, 62:686-690. 10.1002/10970142(19880815)62:4<686::AID-CNCR2820620407>3.0.CO;2-7

25. Naguib M, Caceres M, Thomas CR, Herman TS, Eng TY: Radiation treatment of recurrent pheochromocytoma of the bladder case report and review of literature. Am J Clin Oncol. 2002, 25:42-4.

26. Fishbein L, Bonner L, Torigian DA, Nathanson KL, Cohen DL, Pryma D, Cengel KA: External beam radiation therapy EBRT for patients with malignant pheochromocytoma and non-head and -neck paraganglioma: combination with 131I-MIBG. Horm Metab Res. 2012, 44:405-410. 10.1055/s-0032-1308992

27. Gottfried ON, Liu JK, Couldwell WT: Comparison of radiosurgery and conventional surgery for the treatment of glomus jugulare tumors. Neurosurg Focus. 2004, 17:4.

10.3171/foc.2004.17.2.4

28. Pollock BE: Stereotactic radiosurgery in patients with glomus jugulare tumors . Neurosurg Focus. 2004, 17:10. 10.3171/foc.2004.17.2.10 